Helgoländer wiss. Meeresunters. 19, 58-67 (1969)

\title{
Dissodinium pseudocalani sp. nov., ein parasitischer Dinoflagellat auf Copepodeneiern
}

\author{
G. DREBES \\ Biologische Anstalt Helgoland, Meeresstation, Helgoland
}

\begin{abstract}
Dissodinium pseudocalani sp. nov., a parasitic dinoflagellate on copepod eggs. In spring 1967 and 1968, a new marine dinoflagellate species, Dissodinium pseudocalani, was discovered off Helgoland in the German Bight. The species lives as ectoparasite on eggs of the copepod Psendocalanus elongatus. The vesicular parasitic stage adheres to the host by a suction organ. After the substrate of the egg is consumed, the parasite develops into a spherical primary cyst which produces 8,16 or 32 oval secondary cysts by simultaneous cell divisions. Each secondary cyst forms 16 or 32 dinospores. The dinospores are colourless, flagellated and of the Gymnodinium-type. During reproduction a large reddish or greenish coloured food source is resorbed by the protoplast and diminishes in size. In regard to its mode of reproduction, Dissodinitum pseudocalani is related to D. lunula and the parasitic species of Chytriodinium.
\end{abstract}

\section{EINLEITUNG}

Unsere Kenntnis von parasitischen Peridineen beruht im wesentlichen auf Untersuchungen im westlichen Mittelmeer (Dogiel 1906, Chatton 1920, Cachon 1964). Uber ihr Vorkommen in der Nord- und Ostsee ist wenig bekannt. Fast 40 Jahre sind vergangen, seit ReIchenow (1930) sein Kapitel "Parasitische Peridinea" mit folgendem Ausblick abschloß: „Da eine gründliche Untersuchung der parasitischen Peridineen in der Nord- und Ostsee noch aussteht, mußten in der systematischen Ubersicht zahlreiche Formen Erwähnung finden, die in unserem Gebiet bisher nicht nachgewiesen sind. Denn es werden sicher manche von ihnen zur Beobachtung kommen, wenn bei Planktonuntersuchungen die Aufmerksamkeit auf sie gerichtet wird. Jeder Befund in dieser Richtung ist also wertvoll. Bei den meisten bedarf auch die Entwicklungsgeschichte noch weiterer Erforschung. Selbst bei den bestbekannten sind wir über das Schicksal der Schwärmer und die Art und Weise der Neuinfektionen noch gar nicht unterrichtet."

An dieser Situation hat sich bis heute nichts geändert, und auch seine Vorhersage ist eingetroffen. Bei Routineuntersuchungen des Helgoländer Phytoplanktons wurde eine parasitische Peridinee auf Eiern des Copepoden Pseudocalanus elongatus entdeckt. Wegen des seltenen und jahreszeitlich begrenzten Auftretens des Parasiten sind die mitgeteilten Ergebnisse noch unvollständig. Sie sollen bei nächster Gelegenheit mit Studien 
zur Zytologie und den Primärvorgängen bei der Infektion des Wirtes ergänzt werden. Der Parasit wird hier provisorisch zu der Gattung Dissodinium gestellt.

D i a g o s e: Dissodinium pseudocalani sp. nov. Marine Peridinee, zuerst ektoparasitisch auf Eiern des Copepoden Pseudocalanus elongatus, später frei im Plankton treibend. Parasitisches Stadium blasenförmig, mit Saug-Haftorgan in der Wirtszelle verankert. Saug-Haftorgan knopfartig, mit netzartig verbundenen Leisten. Primärzyste kugelförmig. 151-250 $\mu$ Durchmesser. Protoplast mit großem, rötlichen oder grünlichen Nahrungsballen. Durch simultane Teilung Bildung von 8,16 oder 32 Sekundärzysten, oval, durchschnittlich $76 \times 47 \mu$ groß. In der Sekundärzyste entstehen durch sukzedane Teilung 16 oder 32 farblose begeißelte Dinosporen, durchschnittlich 15,4 $\times$ 12,6 $\mu$ groß, Gymnodinium-Typ. Fundort Reede von Helgoland, April-Juni.

Diag nos is: Peridinea marina, primum modo ectoparasitico affixa ad ova Copepodi Pseudocalani elongati, deinde in planctone fluitans soluta. Dum parasitus forma vesica inhaerens in cella hospitis instrumento simili ancora quae est forma bulla cum regulis coniunctis similibus reti. Cystis primarius globosus, diametro 151-250 $\mu$. Magnum corpus alimenti rubens vel viridis est inclusum in protoplastum. 8, 16 vel 32 cystes secundarii procreantur uno tempore, ovales forma, circa $76 \times 47 \mu$ magnitudine. In cysti secundario per divisionem successivam 16 vel 32 dinosporae incoloratae flagellatae nascuntur, circa $15,4 \times 12,6 \mu$ magnitudine, typo Gymnodinii. Locus repertionis mare quod est circum insulam Helgolandiam in tempore Aprilis usque ad Junium.

\section{MATERIAL UND METHODE}

Das Untersuchungsmaterial stammt aus dem Plankton der Deutschen Bucht in der südlichen Nordsee. Untersucht wurden Planktonfänge, welche werktäglich gegen 9 Uhr vormittags unabhängig von der Richtung des Gezeitenstroms von dem Forschungsboot „Ellenbogen “ der Biologischen Anstalt Helgoland eingebracht werden. Es handelt sich um $75 \mu$-Netzfänge aus dem Oberflächenwasser der Reede zwischen der Insel Helgoland und der ihr östlich vorgelagerten $D$ üne.

Parasiten in den verschiedensten Entwidklungsstadien wurden unter dem Stereomikroskop mit einer Mundpipette isoliert, sodann gemessen und fotografiert. Kulturversuche schlugen fehl, doch konnte die kurzfristige Weiterentwicklung einiger Individuen im Lichtthermostaten bei $7^{\circ} \mathrm{C}$ oder einem Raum von $15^{\circ} \mathrm{C}$ beobachtet werden.

\section{ERGEBNISSE}

\section{Vorkommen in der Natur}

Mit der Frühjahrsblüte der Planktonalgen stellt sich auch der Parasit Dissodinium pseudocalani ein. Zum ersten Male wurde er am 25. April 1967 gefunden und auch noch im Mai 1967 beobachtet. Im folgenden Jahre war er vom 18. April bis 7. Juni in den Planktonfängen zu finden. In diesem Zeitraum stieg die Temperatur von $6,2^{\circ} \mathrm{C}$ 


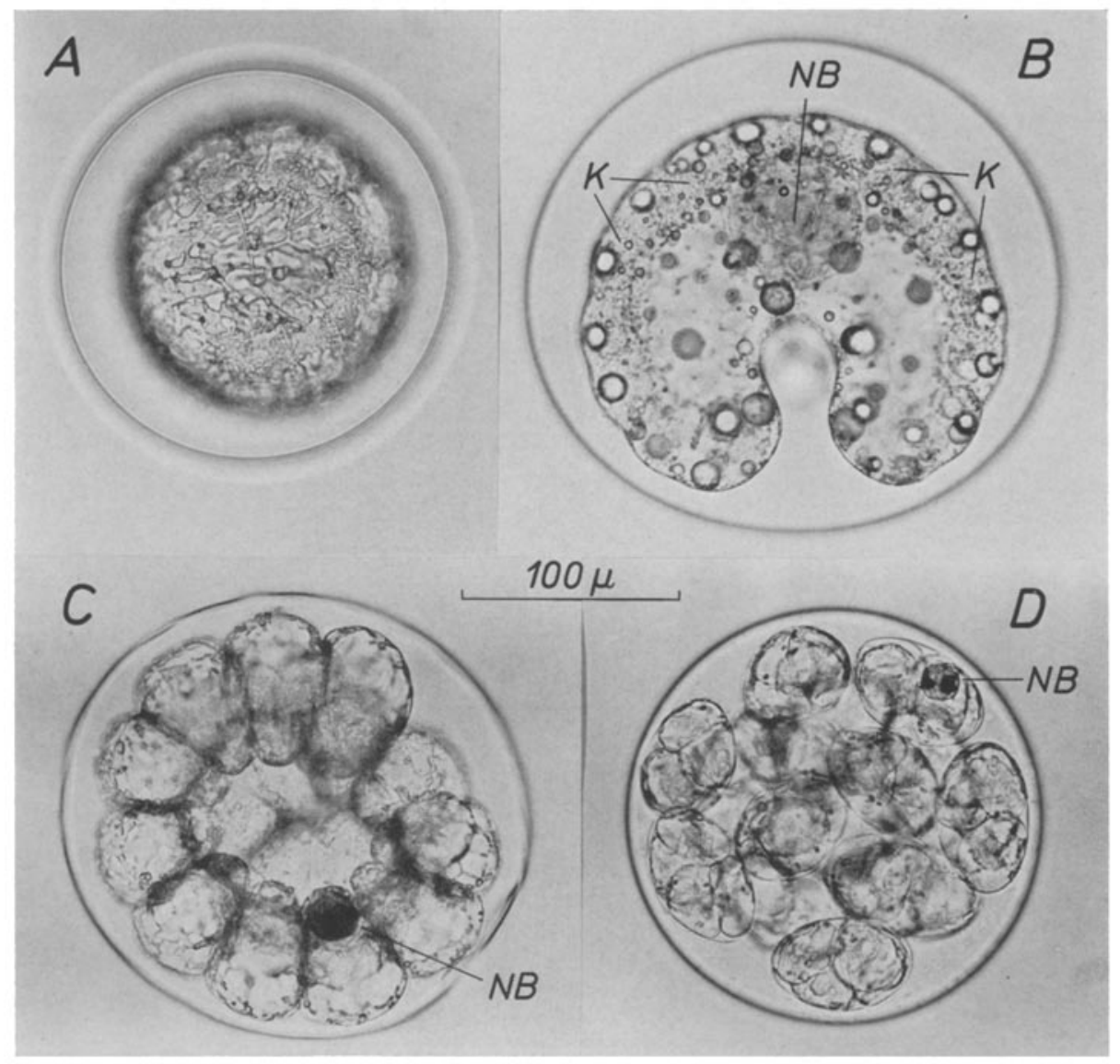

Abb. 1: Dissodinium pseudocalani. A Junge, vom Copepodenei abgelöste, im Plankton treibende Primärzyste. $B$ Eingekrümmter Protoplast mit zahlreichen Kernen $(K)$. Sie sind in der äußeren Zone aneinandergereiht; in Zentrum der Zelle ein großer orangefarbener Nahrungsballen (NB). C Der spiralig gewundene Protoplast hat sich simultan in 16 Segmente aufgeteilt, von denen eines den kleiner gewordenen, leuditend roten Nahrungsballen (NB) enthält. $D$ Zyste mit 16 ovalen, bereits vierzelligen Sekundärzysten. NB, Nahrungsballen

auf $11,6^{\circ} \mathrm{C}$, dabei wechselte ein zunächst reines Diatomeen-Plankton in ein heterotrophes Peridineen- und Zooplankton über. Einer Massenentwicklung von Coscinodiscus concinnus folgte eine ebenso starke von Chaetoceros socialis. Anfang Mai wurde die Diatomeenblüte von heterotrophen Peridinium-Arten, besonders von $P$. depressum und $P$. pallidum, völlig verdrängt. Durch die Menge von $P$. depressum waren die Planktonproben tief-rosarot gefärbt. Hierzu gesellten sich zahlreiche Copepoden, Polychaetenlarven und vereinzelt Sagitta. Auf dem Höhepunkt dieser Entwicklung - während der zweiten Maihälfte - konnte das Untersuchungsobjekt relativ leicht gefunden werden. Nicht selten waren auch Dissodininm lunula, dessen Parasitennatur noch umstritten ist (siehe Diskussion), und Oodinium pouchetii, eine als Ektoparasit auf dem Tunikaten Oikopleura dioica lebende Peridinee. Sie wurde bisher im Mittelmeer und an der fran- 


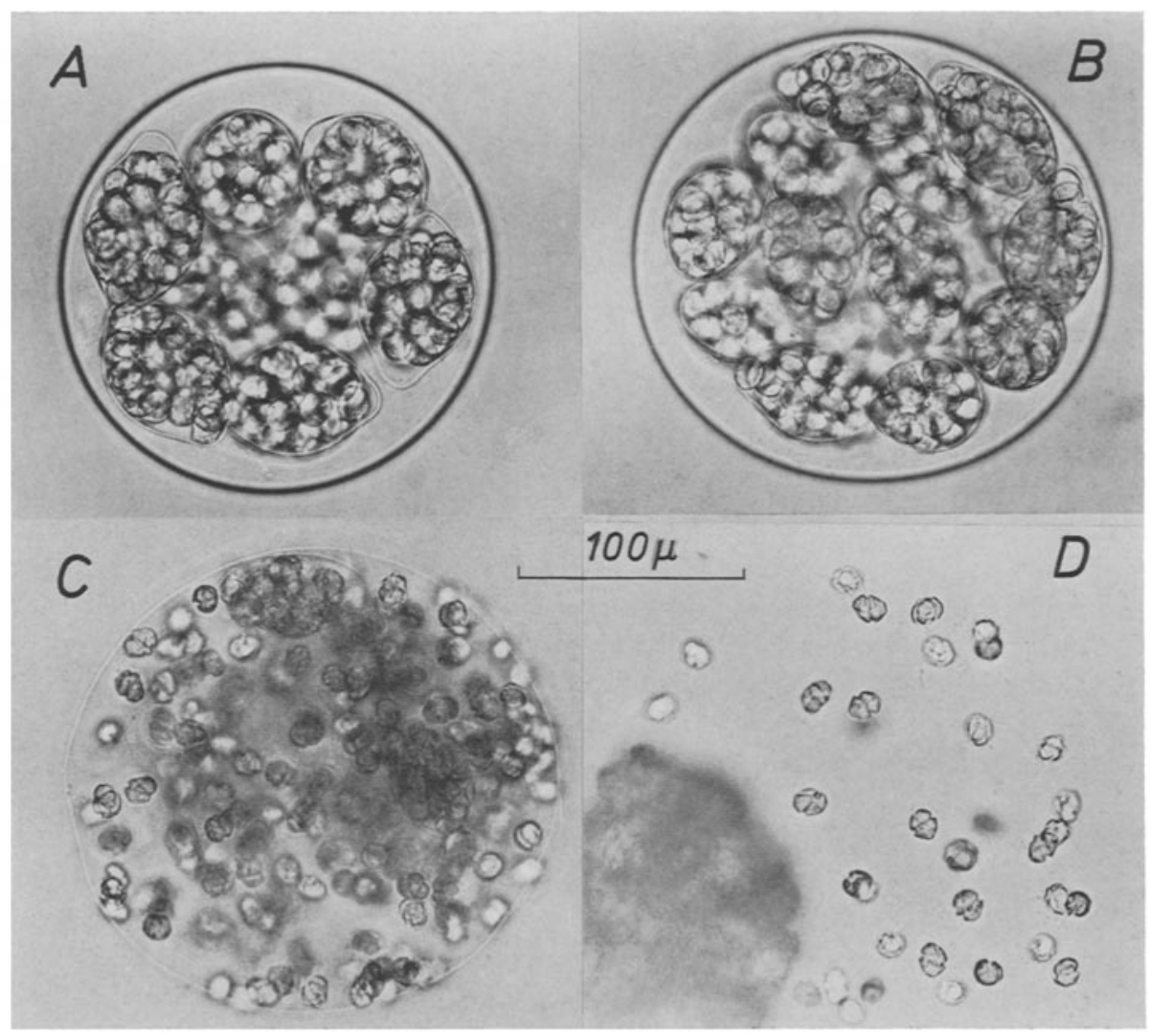

Abb. 2: Dissodinium pseudocalani. A Zyste mit acht Sekundärzysten, je 32 Dinosporen enthaltend. $B$ Zyste mit 16 Sekundärzysten. $C$ Entleerung der Sekundärzysten, die Dinosporen schwimmen frei in der Zyste. $D$ Austritt der Dinosporen aus der Zyste

zösischen Westküste beobachtet, ihr Vorkommen ist nun auch in der Nordsee nachgewiesen. Dissodinium lunula und Oodinium pouchetii werden im Frihjahr, Sommer und Herbst im Plankton angetroffen, während das auf Copepodeneiern schmarotzende Dissodinium pseudocalani lediglich während kurzer Zeit im Frühjahr in Erscheinung tritt. Die Ursachen für diese jahreszeitliche Beschränkung sind noch nicht hinreichend bekannt.

\section{Morphologie und Vermehrungszyklus}

Ahnlich wie bei Dissodinium lunula verläuft die Entwicklung des Ektoparasiten D. pseudocalani von einer Primärzyste über Sekundärzysten zur Ausbildung begeißelter Dinosporen. Die Primärzysten treiben in der Regel losgelöst von ihrem Wirt, den Eiern von Pseudocalanus elongatus, in verschiedenen Stadien ihrer Entwicklung im Plankton (Abb. 1 und 2). Sie sind annähernd kugelförmig, seltener leicht oval. Bei 55 
gemessenen Primärzysten schwankte der Durchmesser zwischen 151 und $250 \mu$. Wie Abbildung $1 \mathrm{~A}$ zeigt, wird das Lumen der Zyste nur zum Teil von dem abgekugelten Protoplasten ausgefüllt. Er schimmert grünlich oder orangefarben, entspricht also der Farbe des Wirtssubstrates, der Copepodeneier. Der an der Peripherie liegende Kern hat sich in diesem Stadium meist schon ein-bis zweimal geteilt. Zytokinesen bleiben zunächst aus, der Protoplast krümmt sich jedoch nach weiteren Mitosen ein (Abb. $1 B$ ). Die Kerne liegen nicht regellos im Plasma verteilt, sondern sind in dem sich wurmartig verlängernden Protoplasten perlschnurartig aneinandergereiht. In Abbildung $1 B$ zieht sich die Kernreihe an der äußeren Krümmungslinie entlang. Neben lichtbrechenden Einschlüssen fällt in den meisten Fällen ein großer orangefarbener Ballen ins Auge. Es dürfte sich um einen "Nahrungsballen" handeln, der im Laufe der weiteren Entwicklung resorbiert und kleiner wird (Abb. $1 B-D$ ). In Abbildung $1 \mathrm{C}$ hat sich der wachsende Protoplast zu einer Spirale verlängert, bevor er simultan in 16 Segmente aufgeteilt wurde. Eines der Segmente enthält den inzwischen stark zusammengeschrumpften Nahrungsballen. Dieser nun meist leuchtend rot gefärbte "Augenfleck" erleichtert sehr das Auffinden solcher und späterer Entwicklungsstadien in den Planktonfängen. Vielleicht wurden Stadien mit grünstichigem Nahrungsballen leichter übersehen und daher nur seltener isoliert.

In Abbildung $1 \mathrm{D}$ haben sich die 16 Segmente voneinander gelöst und die ovale Form der Sekundärzysten angenommen. Sie sind im Mittel $76 \times 47 \mu$ groß. Die Anzahl der Sekundärzysten in einer Zyste ist nicht konstant (Abb. 2 A, B). Von 279 aus dem Plankton isolierten Zysten enthielten 20 je 8,207 je 16 und 52 je 32 Sekundärzysten. Es iberwiegt bei weitem die Anzahl 16. Entsprechende Schwankungen wurden auch für Dissodinium lunula und Chytriodinium (APstern 1906, Dogrel 1906) angegeben. Die Aufteilung des Zellinhaltes der Sekundärzysten zu Dinosporen verläuft sukzedan. Abbildung $1 \mathrm{D}$ zeigt eine Zyste mit 16 Sekundärzysten im 4-Zellenstadium. In jeder Sekundärzyste entstehen 16 oder 32 begeißelte Schwärmer (Abb. 2). Die farblosen, im Mittel 15,4 × 12,6 $\mu$ großen Dinosporen gehören dem Gymnodinium-Typ an (Abb. $2 \mathrm{C}$ und $D$ ). In einer der zahlreichen Dinosporen kann man häufig noch den inzwischen winzigen Nahrungsballen finden. Das weitere Schicksal der Dinosporen kann bisher nur erschlossen werden, da direkte Beobachtungen fehlen.

\section{Wirt und Infektion}

Erst gegen Ende der Beobachtungszeit konnte die Parasitennatur von Dissodinizm pseudocalani nachgewiesen werden. Die Peridinee ernährt sich zuerst als Ektoparasit von den Eiern des Copepoden Pseudocalanus elongatus (Abb. 3 und 4). P. elongatus legt seine grünlichen, seltener rötlich gefärbten Eier in großen zusammenhängenden Klumpen ab (Abb. 3 A). Die Eier lösen sich leicht von dem Tier und treiben im Verband oder einzeln im Plankton. Vor ihrer Entwidklung zu Nauplien werden sie von den Dinosporen befallen. Die Infektion kann bereits erfolgen, solange die Eier noch am Tier angeheftet sind (Abb. $3 B$ ). Offenbar setzt sich die bewegliche Dinospore auf der Eioberfläche fest, bildet ein Saug-Haftorgan aus (Abb. $4 B-D$ ) und zehrt den Inhalt des Eies unter starkem Heranwachsen auf, wie die direkte Beobachtung in den Abbil- 


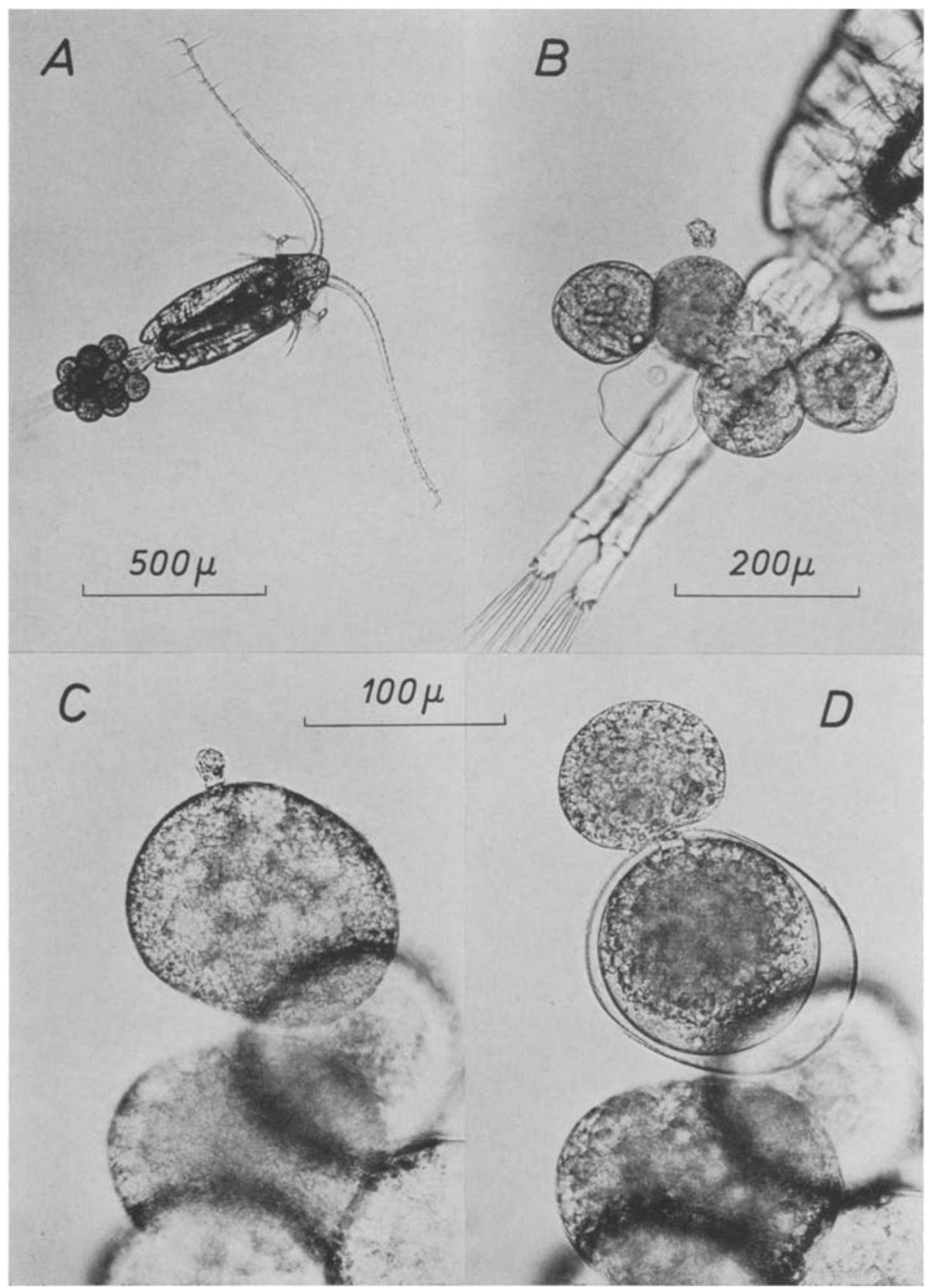

Abb. 3: Dissodinium psendocalani. A Der Copepode Pseudocalanus elongatus mit Eigelege. $B Z$ wei der fünf an einem Tier angehefteten Eier sind infiziert. Oben ein Anfangsstadium, der Parasit hat gerade mit dem Aussaugen eines Eies begonnen; darunter eine Eihülle mit dem in ihr verbliebenen Saug-Haftorgan des Parasiten. C, D Im Abstand von etwa 20 Stunden aufgenommene Infektionsstadien 


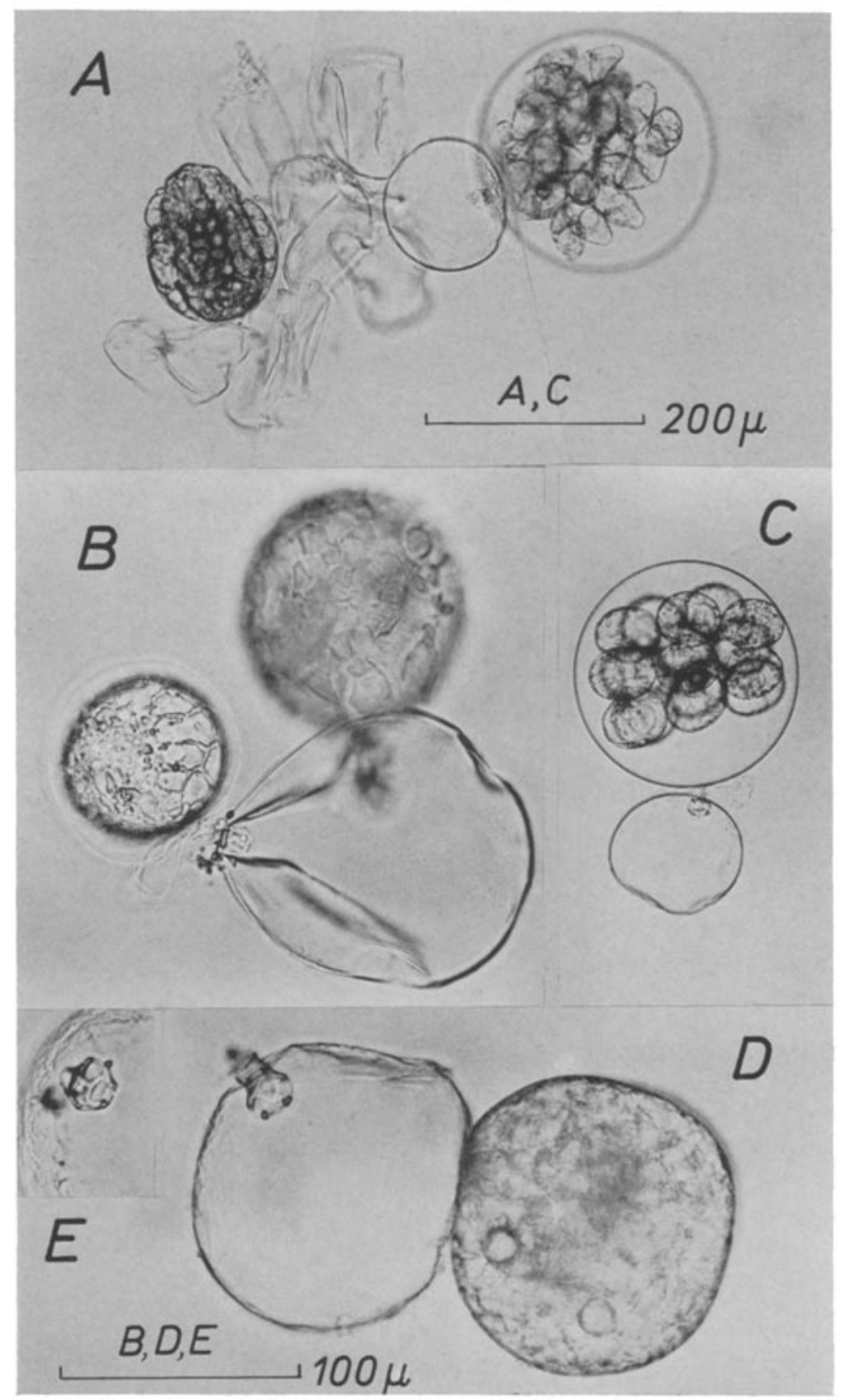

Abb. 4: Dissodinium psetdocalani. A Eihüllen und Nauplius von Pseudocalanus elongatus sowie einer im Stadium der Sekundärzysten mit dem leeren Wirtsei verbundenen Peridinee. $B$ Doppelinfektion eines Eies; der kleinere Parasit scheidet die Membran der Primärzyste $a b$. $C$ Parasit mit 16 Sekundärzysten, das Saug-Haftorgan ist in der leeren Eihülle sichtbar. $D, E$ Eihülle mit Saug-Haftorgan des Parasiten, seitlich und in schräger Aufsicht. Netzartig verbundene Leisten geben dem Organ Festigkeit. Redits ein noch intaktes Ei von Pseudocalanus elongatus 
dungen $3 C$ und $D$ zeigt. Zwischen diesen Aufnahmen liegen etwa 20 Stunden; leider ging die Peridinee dann zugrunde. Ihre weitere Entwicklung dürfte jedoch entsprechend der Darstellung in Abbildung $4 B$ verlaufen. Dieses $\mathrm{Ei}$ ist ausnahmsweise von zwei Dinosporen infiziert worden. Nachdem die beiden Parasiten seinen Inhalt ausgesogen haben, wandeln sie sich unter Bildung einer vom Protoplasten sich abhebenden Membran (Abb. $4 B$ links) in Primärzysten um. Im allgemeinen trennt sich die Primärzyste von der Eihülle und vollendet, wie bereits geschildert, ihre Entwicklung frei im Plankton. Nur selten wurden spätere Entwicklungsstadien des Parasiten in Verbindung mit leeren Eihüllen angetroffen (Abb. $4 A$ und $C$ ); solche Funde ermöglichten es, näheren Aufschluß über das Saug-Haftorgan zu erhalten, das in dem leeren Ei zurückbleibt (Abb. $3 B, 4 A-D$ ). Es mündet nach außen in einen schlanken Hals, während es sich in das Eiinnere knopfartig erweitert. Das Organ ist durch netzartig verbundene Leisten stabilisiert (Abb. $4 D, E$ ) und sichert das Haften des Ektoparasiten an dem Ei, bis dessen Inhalt aufgesogen ist.

Es sind über ein Dutzend Infektionen an den Eiern von Pseudocalanus elongatus festgestellt worden, dagegen nicht eine einzige Infektion an gleichzeitig vorhandenen Eiern anderer Copepodenarten. Eine Aussage über die Spezifität des Wirts soll damit aber noch nicht gemacht sein.

\section{DISKUSSION}

Die Beobachtungen an Dissodinium pseudocalani, einer neuen parasitischen Peridinee in der Nordsee, knüpfen an die Untersuchungen von Dogres (1906) an. Dieser konnte in Neapel von Anfang Mai bis Mitte Juli 1905 den Entwicklungszyklus von D. lunula annähernd aufklären, außerdem drei neue, auf Copepodeneiern parasitierende Arten beschreiben, die CHATToN (1912) seiner neuen Gattung Cbytriodinium einordnete. Alle von DogIEL untersuchten Arten stimmen in ihrer Entwicklung darin überein, daß sich in einer primären Zyste Sekundärzysten differenzieren, in denen dann begeißelte Dinosporen vom Gymnodinium-Typ ausgebildet werden. In gleicher Weise verhält sich auch Dissodinium pseudocalani. Mit Ausnahme von $D$. lunula ist für alle diese Formen eine parasitische Phase zwischen Dinospore und Primärzyste nachgewiesen worden. Das Schicksal der Dinosporen von $D$. lunula ist unbekannt, jedoch vermutet von STOSCH (1967) auch bei dieser Art ein parasitisches Stadium. Die Primärzysten sind anfangs nahezu farblos, sie ergrünen erst während der Bildung der Sekundärzysten und Dinosporen. Die Dinosporen bleiben in Kultur nach eigenen Beobachtungen wochenlang am Leben, ohne sich weiterzuentwickeln. Sie dürtten also „den durch Photosynthese motorisch autonom gemachten Schwärmzustand eines Parasiten darstellen, dessen Wirt freilich unbekannt ist" (voN Sтоsсн 1967, p. 627). In der Untersuchung von SwIFT \& TAYLOR (1967) an Pyrocystis (Dissodinium) lunula aus subtropischen Gewässern des Atlantischen Ozeans werden diese Fragen nicht erörtert. Frische Kulturen enthielten beträchtliche Mengen Schwärmer und relativ wenige Sekundärzysten. In alternden Kulturen sank die Anzahl der Schwärmer, während gleichzeitig die der Sekundärzysten anstieg. Nach Uberführung in frische Nährlösung dominierten wieder die Schwärmer. Unerklärlich bleibt, daß der Anteil der Primär- 
zysten in den Kulturen nur selten 0,5\% überstieg. Der Obergang vom Schwärmer zur Primärzyste ist bei diesem Objekt nicht beobachtet worden, der Entwicklungszyklus ist auch in diesem Falle nicht vollständig bekannt. Zweifel an der Identität des von SWIFT \& TAYLOR untersuchten Materials mit Dissodinium lunula sind nicht ausgeschlossen, nachdem schon Lebour (1925) darauf hingewiesen hat, daß sich unter der Bezeichnung $D$. lunula möglicherweise verschiedene Formen verbergen könnten.

Auch die Beobachtungen von Dogrel (1906) über den Entwicklungszyklus der Chytriodinium-Arten sind lückenhaft und bisher niemals ergänzt worden. Über die Herkunt der Copepodeneier und ihre Infektion wurden keine Angaben gemacht. Auch der Entwicklungsablauf von der Primärzyste zu den Dinosporen läßt noch manche Frage offen.

Die weitgehende Übereinstimmung mit dem Entwicklungsgang von Dissodinium lunula läßt die provisorische Eingliederung des Untersuchungsobjektes in der Gattung Dissodinium vertretbar erscheinen, solange uns die Biologie der hier diskutierten Formen nicht näher bekannt ist.

\section{ZUSAMMENFASSUNG}

1. Dissodinium pseudocalani ist eine neue, auf den Eiern des Copepoden Pseudocalanus elongatus ektoparasitisch lebende Peridinee. Die Art tritt im Plankton der Deutschen Bucht von Mitte April bis Anfang Juni auf.

2. Anfänglich ist der blasenförmige Parasit mit einem stabilen Saug-Haftorgan in seiner Wirtszelle verankert. Nachdem der Inhalt des Eies aufgenommen ist, löst er sich als kugelförmige Primärzyste von einem Durchmesser zwischen 151-250 $\mu \mathrm{ab}$. Sie enthält einen gefärbten Nahrungsballen, der während der Vermehrungsteilungen zusammenschrumpft.

3. Durch simultane Teilung entstehen in der Primärzyste 8, 16 oder 32 ovale Sekundärzysten, in denen sukzedan jeweils 16 oder 32 begeißelte Dinosporen gebildet werden. Die Dinosporen sind farblos und gehören dem Gymnodinium-Typ an. Die Infektion der Psendocalanus-Eier konnte nicht direkt beobachtet, sondern nur aus den verschiedenen im Plankton gefundenen Entwicklungsstadien erschlossen werden.

Danksagung. Herrn Dr. B. Werner danke ich für seine Hilfe bei der Abfassung der lateinischen Artdiagnose, Fräulein P. Hosumbek und Herrn P. Kanje für ihre Unterstützung bei den fotografischen Arbeiten.

\section{ZITIERTE LITERATUR}

Apstein, C., 1906. Pyrocystis lunula und ihre Fortpflanzung. Wiss. Meeresunters. (Abt. Kiel) 9, 263-269.

Cachon, J., 1964. Contribution à l'étude des Péridiniens parasites. Cytologie. Cycles évolutives. Thèse, Faculté des Sciences de l'Université de Paris, 158 pp. (Sér. A, No 4205; No d'Ordre 5056). 
Chatton, E., 1912. Diagnoses préliminaires de Péridiniens parasites nouveaux. Bull. Soc. zool. Fr. 37, 85-93.

- 1920. Les Péridiniens parasites. Morphologie, reproduction, éthologie. Archs Zool. exp. gén. 59, 1-475.

Dogrel, V., 1906. Beiträge zur Kenntnis der Peridineen. Mitt. zool. Stn. Neapel 18, 1-45.

LeBour, M. V., 1925. The Dinoflagellates of northern seas. The Marine Biological Association of the U.K., Plymouth, 250 pp.

Reichenow, E., 1930. Parasitische Peridinea (einschließlich Ellobiopsidae). Tierwelt N.- u. Ostsee $4(2 \mathrm{~d} 3), 85-100$.

Stosch, H. A. von, 1967. Dinophyta. Handb. PflPhysiol. 18, 626-636.

SWIRT, E. \& TAYLOR, W. R. 1967. Bioluminescence and Chloroplast movement in the dinoflagellate Pyrocystis lunula. J. Phycol. 3, 77-81.

Anschrift des Autors: Dr. G. Drebes

Biologische Anstalt Helgoland

Meeresstation

2192 Helgoland 